\title{
Novel Antibiotics by Late-Stage Derivatization of Marketed Fidaxomicin
}

Chemistry in

Medicine and

Biology

Key words

fidaxomicin

antibiotics

selective

transformation
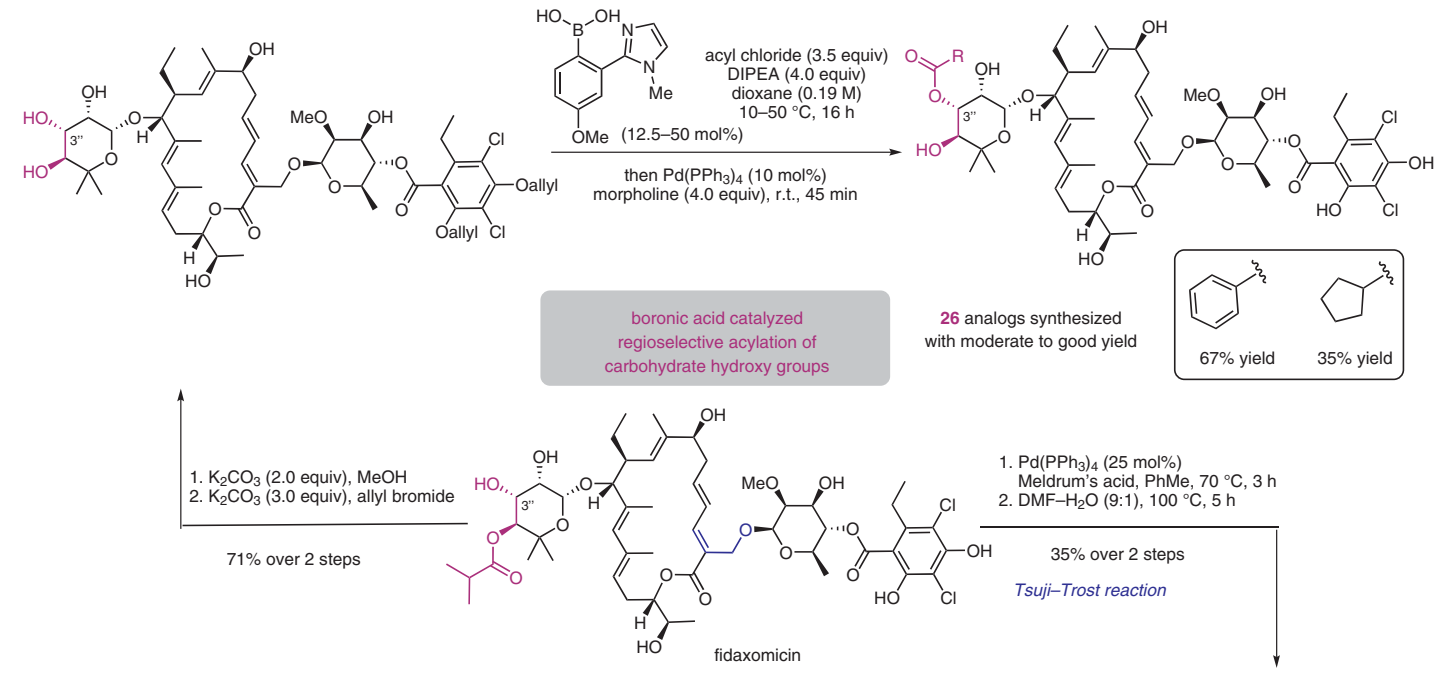

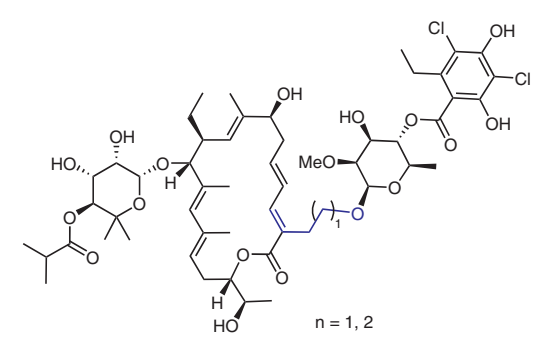

C1- and C2-elongated fidaxomicin
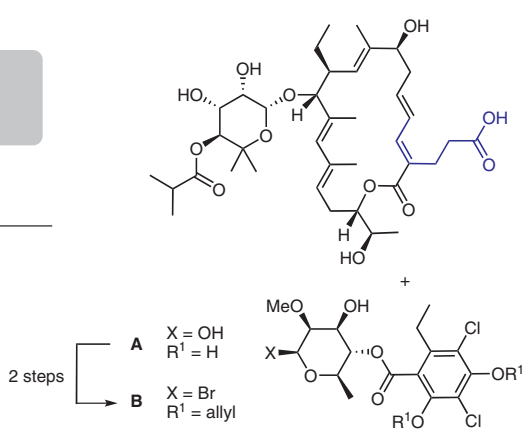

Significance: Fidaxomicin shows antibacterial activity against Gram-positive bacteria. In 2011, fidaxomicin was approved by the FDA for the treatment of $C$. difficile infection, the main cause of hospital-acquired diarrhea. The development of second-generation fidaxomicin derivatives cannot only expand its therapeutic applications, such as the treatment of Mycobacterium tuberculosis, but can be a potential candidate against multidrugresistant bacteria. The authors developed two strategies for the synthesis of novel analogues with promising antibacterial activities directly from fidaxomicin.
Comment: Two strategic, site-selective reactions were used for late-stage functionalization of fidaxomicin. A boronic acid catalyzed regioselective acylation can acylate the $\mathrm{C}^{\prime \prime}$-OH in the presence of six other hydroxy groups, based on previous work (Org. Lett. 2019, 21, 3789). A palladium-catalyzed regioselective allylic alkylation can replace the right part glycose with Meldrum's acid, and further manipulations allow for glycodiversifications at the right side. Considering the complexity of fidaxomicin, the achieved selectivity is quite impressive. 